Journal of Computer Science 8 (8): 1263-1271, 2012

ISSN 1549-3636

(C) 2012 Science Publications

\title{
Minimizing Interference in Wireless Mesh Networks Based Telemedicine System
}

\author{
${ }^{1,2}$ Mohammad Siraj and ${ }^{2}$ Kamalrulnizam Abu Bakar \\ ${ }^{1}$ College of Engineering, \\ King Saud University, P.O. Box 800, Riyadh-11421, Saudi Arabia \\ ${ }^{2}$ Faculty of Computer Science and Information Systems, \\ Universiti Teknologi Malaysia, Malaysia
}

\begin{abstract}
Problem statement: It has been always a challenging task for the researchers to incorporate information technology advancements in the medical profession. In order to guarantee a reliable network infrastructure that supports the employment of wireless technology and mobile devices, a hybrid infrastructure that integrates wireless networks with the traditional wired Local Area Networks (LANs) is needed. The wired network will provide the stability to the network, improve the overall performance of the hybrid network and provide an alternative to the mobile routes, hence increasing the degree of redundancy and reliability. In recent years, Wireless Mesh Networks (WMN) have become more popular than ever. WMN has advantage of robust fault tolerance. Even if some of the mesh nodes are incapable, there exist many other alternative nodes to serve relay. In addition, multihop of WMN can not only spread coverage but also save both cabling cost and human resource. Health care can drastically benefit by incorporating technological advancements, particularly wireless mesh technology. In this study we have presented a telemedicine system for rural and urban scenario based on WMN so that the medical community gets benefited by state of art broadband connectivity. Also in this study we have addressed a critical issue of performance degradation in WMN due to interference and varying load. Approach: For this we have proposed a routing protocol AODV LBIARM. We have incorporated our routing metric Load Balancing Interference Aware Routing Metric (LBIARM) in AODV protocol to minimize the interference and cater for varying traffic load in multi hop WMN based telemedicine system, thereby maximizing network throughput. Results and Conclusion: This protocol is evaluated by simulating in Opnet Modeler 16.1 PL1. We have found that our proposed protocol performs better than AODV WCETT and AODV HOP-COUNT.
\end{abstract}

Key words: IEEE 802.11, Local Area Networks (LANs), LBIARM, telemedicine, Wireless Mesh Networks (WMN)

\section{INTRODUCTION}

Telemedicine system: The word telemedicine is made up of two words. The first word, "tele," means "at a distance," and the second word, "medicine," is derived from the Latin ars medicina, meaning the art of healing. So medicine can be defined as the science and art of healing. It encompasses a variety of health care practices evolved to maintain and restore health by the prevention and treatment of illness. Combining the both telemedicine can be defined as the practice of medicine at a distance. One of the current accepted definitions of telemedicine is as follows.

Telemedicine involves the use of modern information technology, involving two-way interactive audio/video telecommunications, computers and telemetry, to deliver health services to remote patients. It facilitatec information exchange between primary care physicians and specialists at remote distance from each other.

There are two modes of operation for telemedicine: real-time and store-and-forward. In real-time mode, the information, which can be any combination of audio, video, data and images is transmitted to a remote terminal immediately after acquisition, thus allowing real-time interaction between patients and health care personnel. Some examples are consultation with a remote doctor, ambulatory Electrocardiogram (ECG) monitoring and instant transmission of ultrasound images. Because real-time applications involve continuous, time-critical information exchange, high transmission bandwidths are often required, resulting in relatively high operation cost. In store-and-forward mode, the information gathered is analyzed at a remote

Corresponding Author: Mohammad Siraj, College of Engineering., King Saud University, P.O. Box 800, Riyadh-11421, Saudi Arabia 


\section{J. Computer Sci., 8 (8): 1263-1271, 2012}

terminal at a later time, such that less bandwidth is consumed. Diagnosis and clinical management are the main applications of store-and-forward telemedicine. Some examples of these are transfers of previously recorded ECG and Computer Tomography (CT) scans are Remote patient monitoring, Teleconsultation, Teleradiology, Telepathology, Teledermatology, Teleoncology, Telepsychiatry, Telecardiology, Telehomecare, Tele-surgery. Coury et al. (2011) designed a telemedicine system to Connect the University and Teaching Hospitals to Brazil's national research and education $\mathrm{R}$ and $\mathrm{E}$ network through a $1 \mathrm{Gbps}$ City Fast Ring for $\mathrm{R}$ and $\mathrm{E}$ Institutions. At the moment through this system 48 University and Teaching Hospitals operate their Telemedicine Nuclei .They have a network of more than 200 institutions and their specialists participate in 40 Special Interest Groups, with ca. 2-3 sessions every day. Khodambashi et al. (2008) designed a tactile sensor for detection of skin surface morphology which could be used as an assistant device for diagnosis purposes in teledermatology systems.

A conceptual model of a telemedicine system utilizing IEEE 802.11 Mesh network is shown in Fig. 1 below. Broadly it contains three parts: Wide Area Network (WAN), Wired Infrastructure and Multihop Wireless Local Area Network (WLAN's). The Wired Infrastructure connects the WLAN's to Internet. The WLAN consists of various wireless devices such as wireless access pointer, PDA, RFID Read/Write Sensor and biosensors. The sensors can be stationary sensors as well as mobile.

Wireless mesh networks: IEEE 802.11 Multi Hop Wireless Mesh Networks (WMNs) (Akyildiz et al., 2005) is a promising technology, which provides wireless broadband connectivity to the Internet. WMNs are self-forming, self-healing and self-organizing. Their easy configuration and deployment make them an economical, reliable and simple solution that can be implemented anywhere at any time. The end users can use WMN to access the internet by connecting to any of the routers. WMNs have emerged as a fundamental technology for the next generation of Wireless Networking. They are formed by mesh routers and mesh clients as shown in Fig. 2. WMN are being investigated at this moment to support video-related applications such as video streaming, multimedia messaging, teleconference, voice over IP and video telemedicine due to its superior characteristics compared with other wireless standard, including mobility, high data rate and low cost infrastructure. With such infrastructure in place, a number of critical medical problems can be addressed with the help of WMNs and the stability of the infrastructure backbone can be done.

In a wireless mesh network, the capacity is reduced by interference from simultaneous wireless transmissions. Two types of interference affect the throughput of multi-hop wireless networks: intra-flow interference, which is induced between adjacent nodes on the same routing path and interflow interference, which is caused by nodes from neighboring paths. To provide backbone support to the IEEE 802.11 mesh based telemedicine system, it is necessary to reduce interference in WMNs which is a critical issue.

Interference: Interference is an important factor which influences the ability of two linked nodes to reliably communicate with each other at the desired communication rate. Within the scope of 802.11 based wireless networks, we distinguish between two types of interference: radio interference and channel contention interference. Radio interference represents a physical interference that influences the entire spectrum of electromagnetic waves. Physical interference has two distinctive subtypes: destructive interference and constructive interference. Destructive interference is depicted in Fig. 3a where 3 waveforms can be seen: the first waveform on the top (in red) represents message signal, the second waveform in the middle represents an unwanted interference signal and the bottom waveform represent the resulting signal waveform. As it can be observed in Fig. 3a, the destructive interference has a detrimental effect on the transmitted radio signal, which often results in a loss of transmitted data. On the other hand, in the second type of interference i.e., the constructive interference depicted in Fig. 3b (waveforms are in the same order as in Fig. 3a has the converse effect on the signal as it results in an increase of its amplitude. However, the waveforms used in these two examples are synchronized in time, which is very rarely the case in reality. This means that in most of the cases both forms of interference have a detrimental effect on the signal.

The second type of interference i.e., the contention interference is not a physical interference as in the previous cases although it also produces a negative effect. Contention interference stems from the Carrier Sense Multiple Access with Collision Avoidance (CSMA/CA) based MAC layer of 802.11 protocol that defines the behavior of the 802.11 station, which has to wait until a channel is free to commence its transmission. Therefore, the channel may be occupied by transmission from any node that uses the same channel within the communication range. Due to the license free availability of 802.11 band, this issue is even more pronounced in urban areas that contain a large number of 802.11 based networks. Thus, in urban areas all the co-located networks compete for the use of limited number of channels. In addition to the term interference it is important to clearly define two related terms: communication range and interference range. 


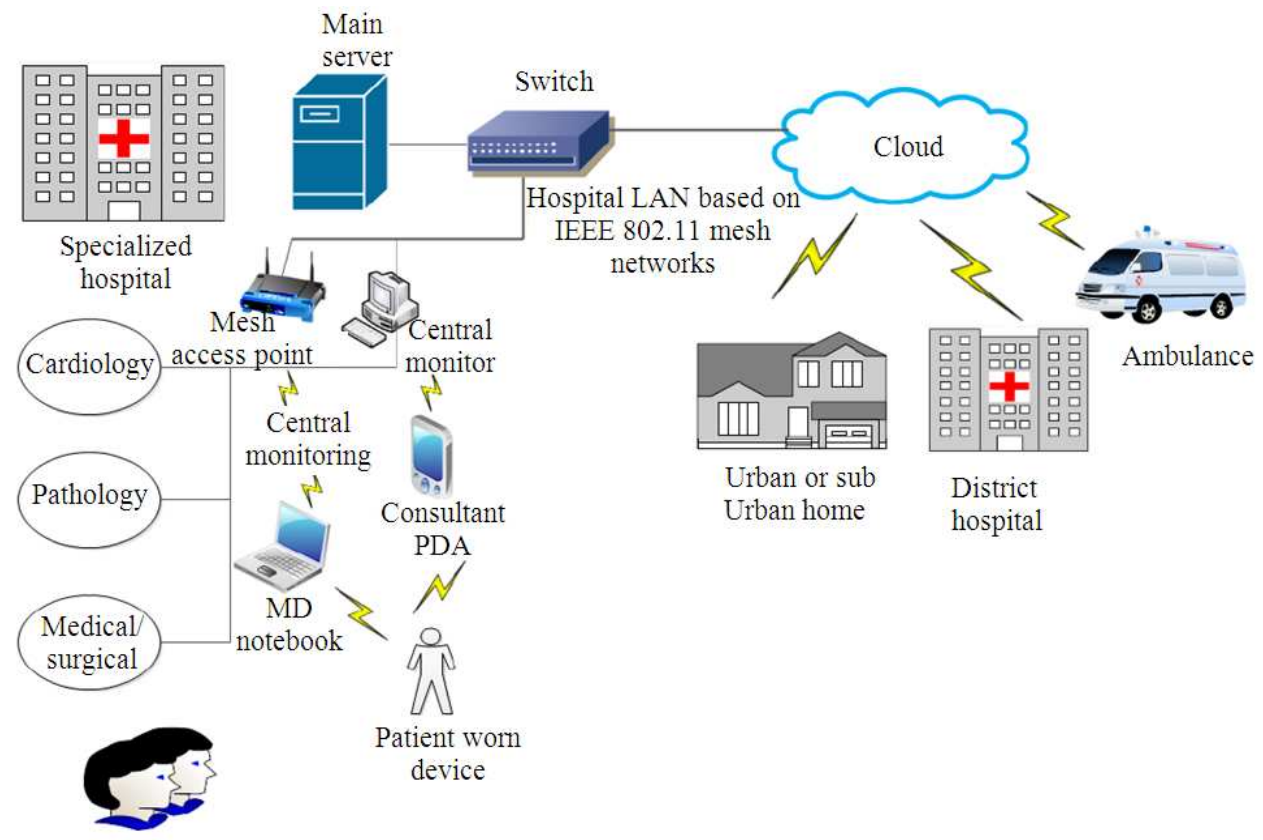

Panel doctors

Fig. 1: Conceptual model of a telemedicine system utilizing IEEE 802.11 Mesh network

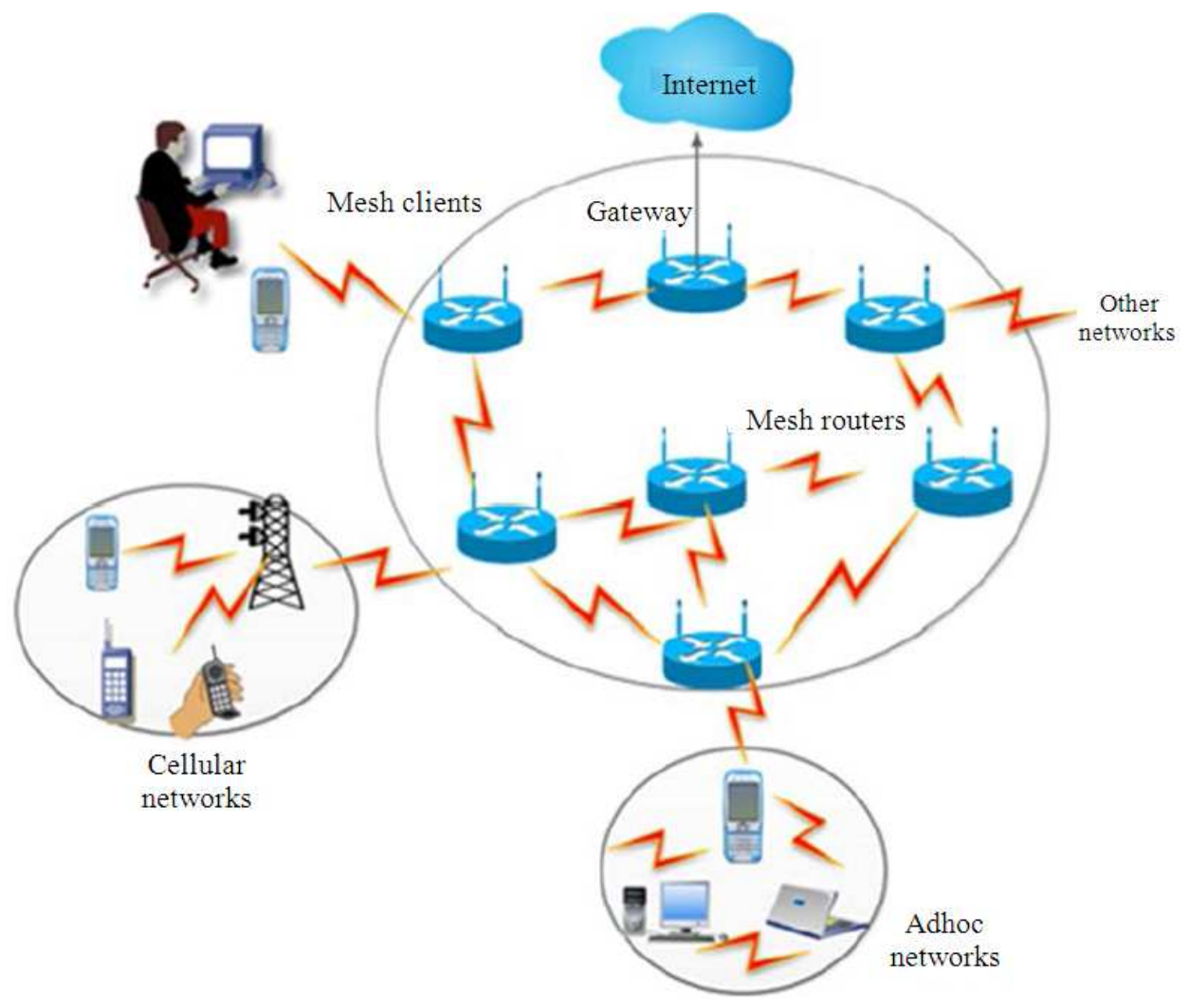

Fig. 2: Wireless Mesh Architecture (Siraj and Bakar, 2011a) 1265 


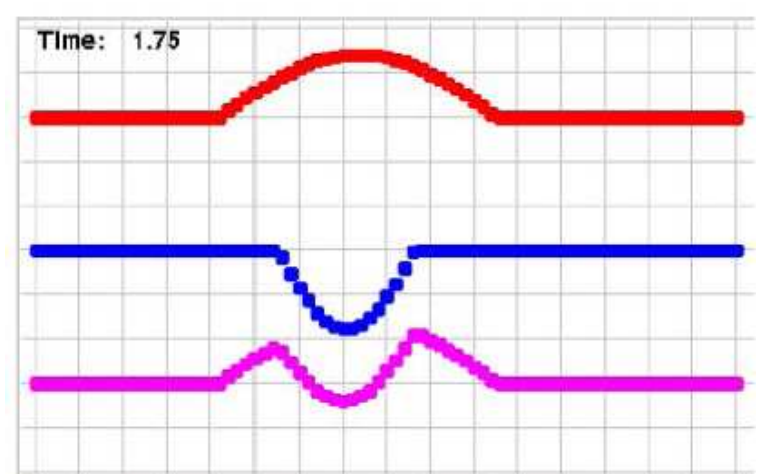

(a)

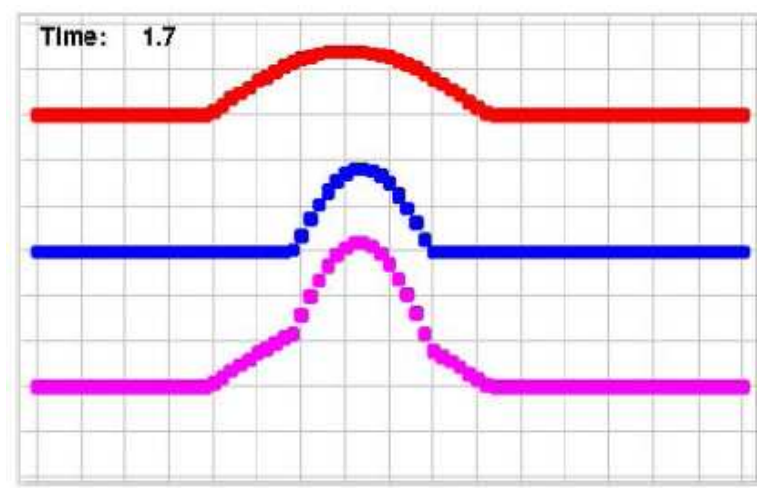

(b)

Fig. 3: Destructive and Constructive Interference (Pradhon and Mirchandani, 2009) (a) Destructive interference (b) Constructive Interference

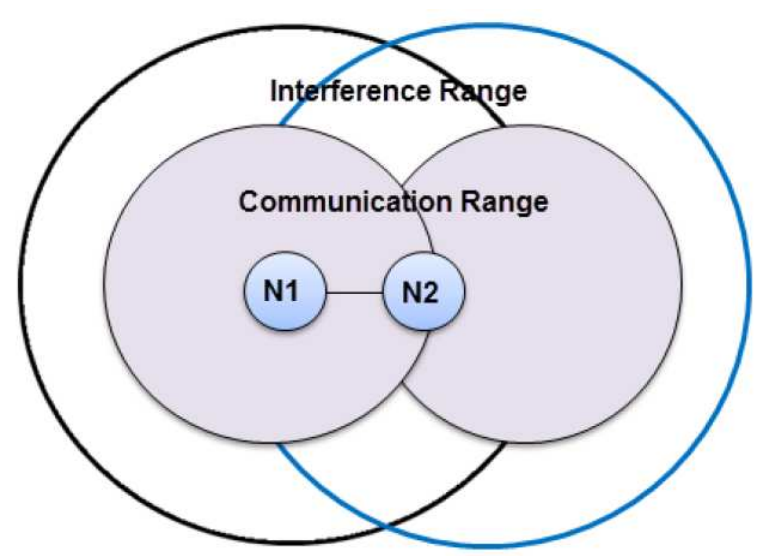

Fig. 4: Communication range and Interference range

Communication range is the range in which a reliable communication between two nodes is possible and interference range is the range in which transmissions from one node can detrimentally affect the transmissions from other nodes on a same or partially overlapping channel. It is important to note that the interference range is always larger than the communication range as shown in Fig. 4.

Contention Interference can further be classified.

Intra flow interference: Intra flow interference occurs due to the adjacent nodes on the same routing path. They compete against each other for channel bandwidth. This intra flow interference causes throughput to degrade severely due to the consumption of the flow bandwidth across each node on the same routing path. The hop count of the flow increases with an increase in end to end delay. This causes congestion. E.g., in Fig. 5, we see the path $1 \rightarrow 2 \rightarrow 3$ causes intra flow interference because of the reuse of channel 1 on the flow $1 \rightarrow 2$ and from $2 \rightarrow 3$. So the path $1 \rightarrow 4 \rightarrow 3$ does not have intra flow interference due to the assigning of two different channels between $1 \rightarrow 4$ and $4 \rightarrow 3$. We can say that that $1 \rightarrow 4 \rightarrow 3$ is a better path in comparison to $1 \rightarrow 2 \rightarrow 3$. So a good intra flow aware interference matric should assign $1 \rightarrow 4 \rightarrow 3$ a lower weight than $1 \rightarrow 2 \rightarrow 3$. In other words we can say that a good routing metric reduces inter flow interference by selecting nonoverlapping channels for adjacent hops of a path.

Inter flow interference: Inter flow interference is the interference occurring due to the other flows operating on the same channels and competing for the medium. This is caused by the multiple flows between different routing paths as shown in Fig. 6. This not only consumes bandwidth of the nodes along its route, it also competes for bandwidth with the nodes following in the neighborhood. In comparison to intra flow interference inter flow interference is harder to control due to the involvement of multiple flows and routes. In Fig. 6 we see inter flow interference due to the two paths namely $1 \rightarrow 4 \rightarrow 3$ and $5 \rightarrow 6 \rightarrow 7$. A good inter flow aware metric should assign a low weight $1 \rightarrow 2 \rightarrow 3$ then to $1 \rightarrow 4 \rightarrow 3$ as path $1 \rightarrow 2 \rightarrow 3$ has less inter flow interference.

Related work: Researchers have tried to integrate telemedicine and telehealth with present day wireless networking technologies. (Zhou et al., 2010) had suggested a telemedicine network based on Wireless Sensor Networks (WSN). Putra et al. (2009) had presented a cross layer design of WLAN for telemedicine system. Morreale (2007) PA had presented an Urban Telehealth network based on Wireless Sensor Network. Shnayder et al. (2005) proposed WSN for Medical care. Malan et al. (2004) had proposed an ad hoc sensor network infrastructure for emergency medical care. Chu and Ganz (2004) had presented a mobile teletrauma system based on $3 \mathrm{G}$ networks. Golmie et al. (2005) presented the performance evaluation of WPANs for sensors and medical application. 

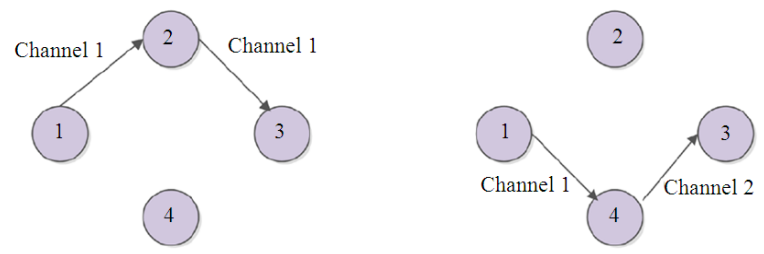

Fig. 5: Intra Flow interference
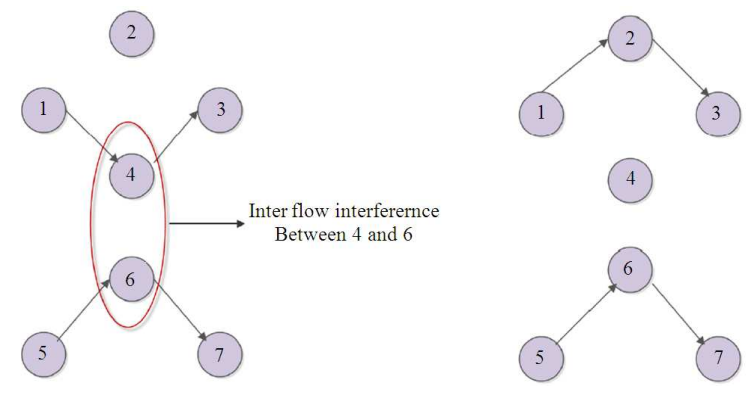

Fig. 6: Inter Flow interference

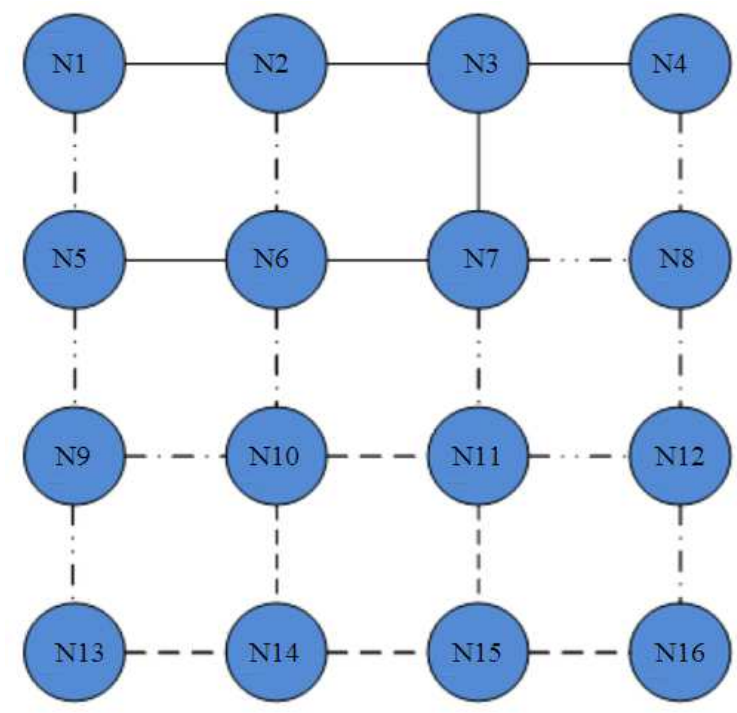

Channel 1

Channel 2

Channel 3 - $-\cdots-$

Channel 4 - - - .

Fig. 7: 4×4 Grid Wireless Mesh Network with 4 channel assignment

Hop count is the most common routing metric used in Dynamic Source Routing (DSR) (Johnson and Maltz, 1996) and Ad-hoc On-demand Distance Vector (AODV) (Perkins et al., 2003) routing protocols. Hop count metric is not suitable for WMN due to parameters like congestion control, scalability and in establishment of paths with maximum throughput. The Expected Transmission Count (ETX) routing metric was proposed by Couto et al. (2003) to address the above mentioned problem. ETX does not take account of load and intra flow interference. It is insensitive to various link rates. The Expected Transmission Time ETT (Draves et al., 2004) routing metric was an improvement over ETX as it took into account the bandwidth of different links. The disadvantage of this metric was it did not consider link load explicitly and as a result was not able to avoid routing traffic through already congested nodes and links. The second disadvantage was it did not minimize intra flow interference as it was not designed for multi radio networks. The Weighted Cumulative Expected Transmission Time (WCETT) (Draves et al., 2004) was designed to improve the ETT metric in the area of multi radio mesh networks by considering channel diversity. A number of routing metrics have been proposed for routing protocols in Wireless Mesh Networks such as metric of interference and channel switching (MIC) (Yaling et al., 2005), Load Aware ETT (LAETT) (Aiache et al., 2008), Exclusive ETT (EETT) (Jiang et al., 2007), Interference Load Aware(ILA) (Shila and Anjali, 2007), interference Aware iAWARE (Subramanian et al., 2006), Adaptive Load Aware Routing Metric (ALARM) (Amir et al., 2009) and a location Aware Routing Metric (ALARM) (Alotaibi and Roy, 2008) were proposed to support load balanced routing and to consider intra and inter-flow interference, in addition to being isotonic. We had found that each routing metric had limitation in not meeting one or more criteria. We had proposed a new routing metric called Load Balancing Interference Aware Routing Metric (LBIARM) (Siraj and Bakar, 2011b) which takes cares of all the above parameters.

AODV LBIARM routing protocol: We incorporate our LBIARM metric in AODV protocol. This protocol finds best route discovery. It selects the route which has minimum interference, high throughput and low end to end delay. The route discovery is based on the following algorithm.

\section{Algorithm for best route discovery:}

Source Node S Broadcasts to Gateway Node G

if (Intermediate Node I receives Route_Request) then

Calculate path load from S to I using the following equation:

$$
\text { LBIARM }=(1-a) \sum_{i \in p} E_{i T}+a \sum_{i \in p} E_{T} T_{i} * N_{i}
$$

Create a Reverse Route to S 


\section{J. Computer Sci., 8 (8): 1263-1271, 2012}

\author{
If Route_Request is duplicate discard \\ Route_Request Packet. \\ if (I is D) \\ unicast Route_Reply to S. \\ else \\ rebroadcast Route_Request \\ endif \\ if (I receives Route_Reply) and (I is not S) \\ then I forward Route_Reply to S \\ else \\ $\mathrm{S}$ updates path load. \\ endif \\ if ( $S$ does not receive Route_Reply for a \\ certain time period $t$ ) then \\ S broadcasts (Route_Request_Error) \\ endif \\ if I receives Route_Request_Error and (I is not G) \\ forward Route_Request_Error. \\ else \\ G updates its links_table. \\ G sends Route_Request to D \\ endif
}

Performance Evaluation:

Simulation environment: The performance evaluation of our proposed metric LBIARM was done with hop count and WCETT metric by incorporating these metric in AODV protocol and simulating in Opnet Modeler 16.1 PL1. The network topology as shown in Fig. 7 was used for simulation. It consists of 16 static mesh routers uniformly randomly placed in a $4 \times 4$ grid in a $800 \times 800$ $\mathrm{m}^{2}$ area. The average distance between each pair of two one hop nodes is the same. The interference range is set to be approximately equal as all mesh routers are with similar transmission powers. The source nodes send Constant Bit Rate (CBR) traffic with UDP as transport protocol, consisting of 1024 byte packets with a sending rate of 20 packets/second. The center mesh router was selected as the Gateway node. The value of was taken as 0.3 for Calculation of WCETT and LBIARM. This value was taken as it was found that optimum value of WCETT is when $\mathrm{a}=0.3$. The following simulation parameters were used (Table 1).

Performance metrics: For evaluation of AODV LBIARM the following metrics were used.

Packet delivery ratio: This is the ratio between the number of data packets successfully received by the destination node and the total number of data packets sent by the source node. This metric reflects the degree of reliability of the routing protocol.
Table 1: Simulation parameters

\begin{tabular}{ll}
\hline Parameter & Value \\
\hline Network scenario & Campus Network \\
Network grid & $800 \times 800$ \\
Number of nodes & 16 \\
Number of radios & 2 \\
Number of channels & 4 \\
Packet size & 1024 \\
Interference range & $400 \mathrm{~m}$ \\
Traffic model & Constant Bit Rate (CBR) \\
Transmission power & $10 \mathrm{~mW}$ \\
Queue size at routers & $50 \mathrm{Kbytes}$ \\
Physical layer protocol & PHY 802.11g \\
CBR sender's rate & 20 packets/sec \\
Transmission rate at physical layer & $54 \mathrm{Mbits} / \mathrm{sec}$ \\
\hline
\end{tabular}

End-to-end delay of data packets: This is the delay between the time at which the data packet originated at the source and the time it reaches the destination and includes all possible delays caused by queuing for transmission at the node, buffering the packet for a detour, retransmission delay at the MAC layer, propagation delay and transmission delay. This metric reflects the quality of routing protocol.

Throughput: This is defined as the amount of data that is transmitted through the network per unit time (i.e., data bytes delivered to their destinations per second).

\section{Simulation results and analysis:}

Packet delivery ratio: Figure 8 shows the percentage of packet delivery ratio between LBIARM and WCETT and HOP-COUNT in presence of interfering traffic. We observe with the increase in flows contention increases. With an increase of interference, the packet loss ratio of all the above metrics also increased. Percentage of Packet delivery also falls down. It is observed AODV LBIARM performance is better than AODV WCETT and AODV.

End to end delay: Figure 9 shows the end to end delay .It is observed AODV LBIARM performs better than AODV WCETT and AODV. This is due to the balancing act of LBIARM which minimizes interference due to the selection of an optimum route. This route is based on less interference taking into consideration of varying traffic load. When the flows are increased it increases the end to end delay. LBIARM improved the end to end delay as AODV HOP-COUNT and AODV WCETT do not balance the traffic flows and create congestion causing large delay. 
J. Computer Sci., 8 (8): 1263-1271, 2012

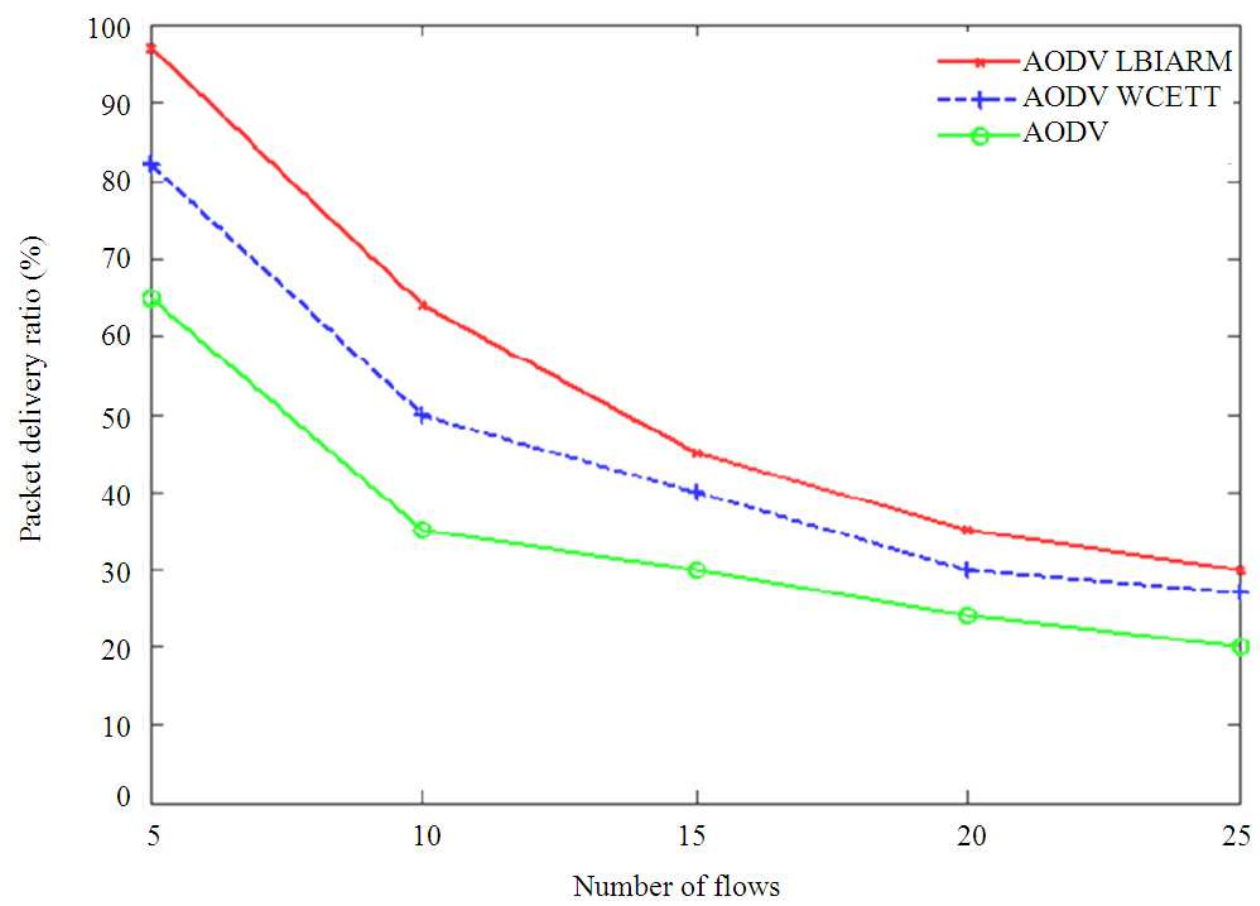

Fig. 8: Packet delivery ratio Vs. number of flows

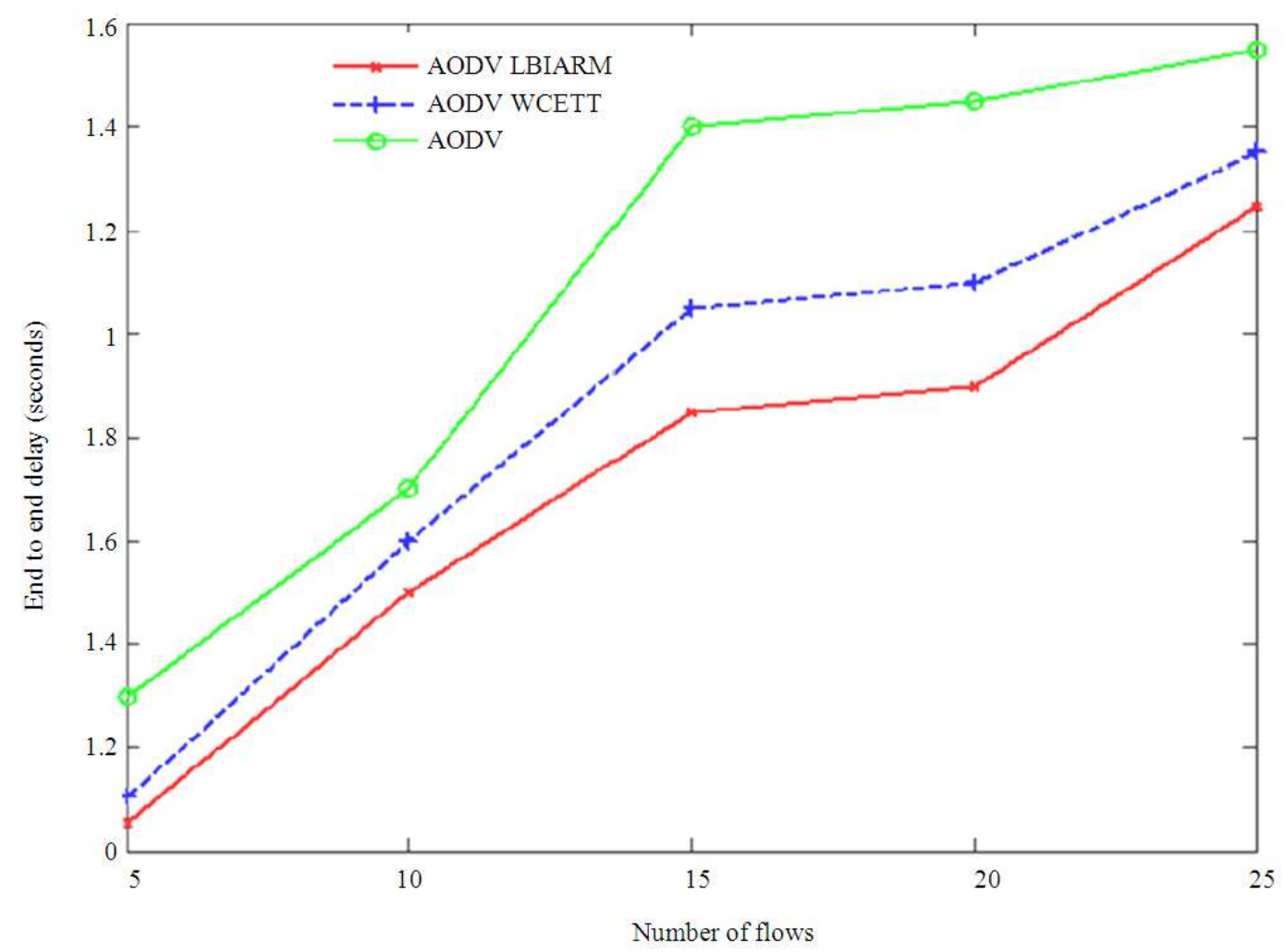

Fig. 9: End to end delay Vs. number of flows

1269 


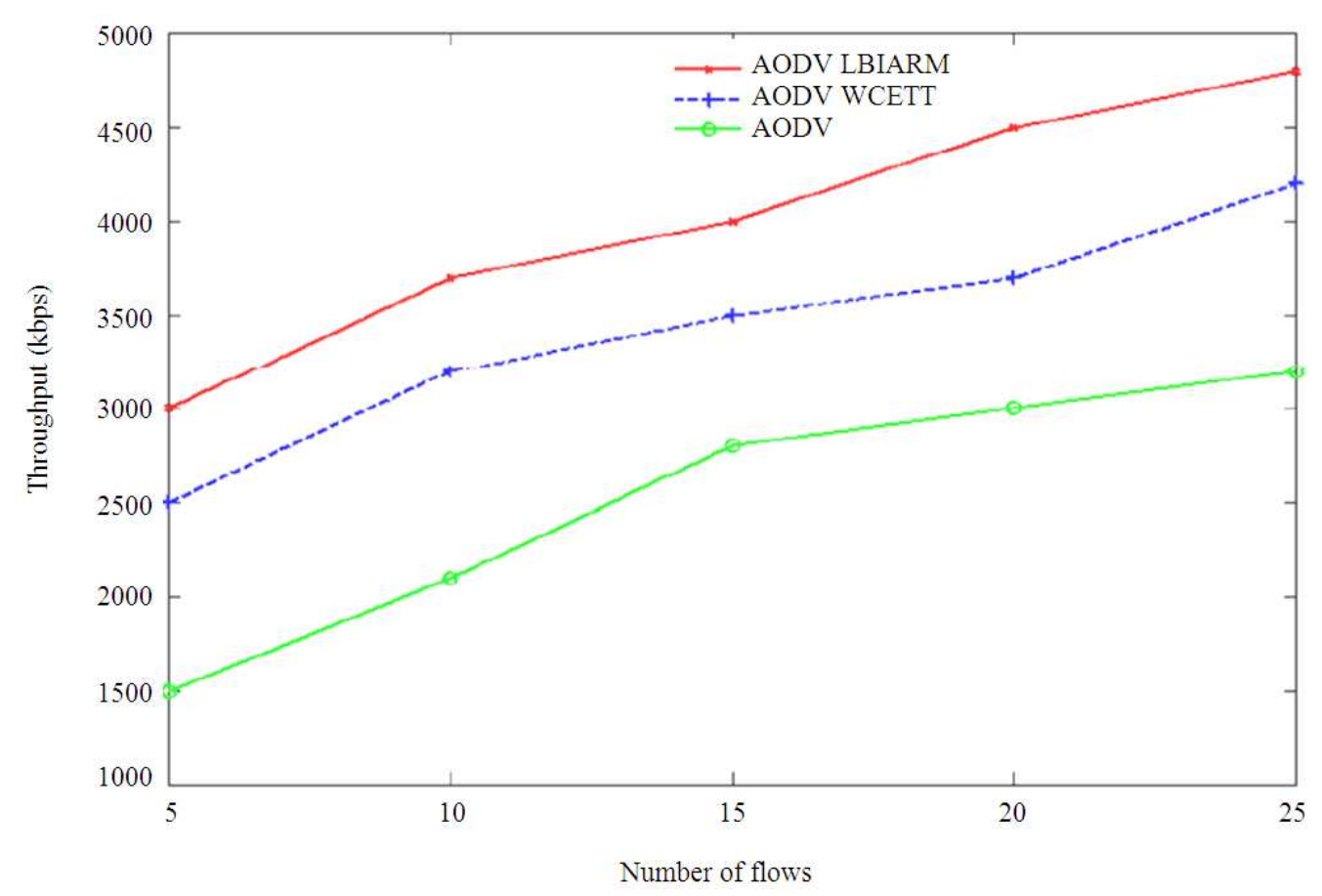

Fig. 10: Throughput (average) Vs. number of flows

Throughput: Figure 10 shows throughput comparison between AODV, AODV WCETT and AODV LBIARM.

The throughput in AODV LBIARM is greater because the other two metrics creates congestion areas. At each hop there is a delay of data packets. AODV LBIARM creates quality links with fewer delays which are less loaded.

\section{CONCLUSION}

In this study we have proposed a telemedicine system which utilizes Wireless Mesh Networks as a backbone to utilize the benefits of Broadband networking in telemedicine. This proposed scenario can be applied to rural as well as for urban areas. A common problem in WMN is the performance degradation with multiple hops. This is due to Interference of the neighbors and varying traffic load. This is a critical issue in WMN. We had incorporated our proposed LBIARM metric in AODV protocol to address this problem. Our metric has the ability to cater for varying load and capture inter and intra flow interference. Also it had the ability to capture difference in transmission rates. We have shown by simulations that AODV protocol incorporated with LBIARM metric performs better as compared to WCETT and HOP-COUNT routing metric. It will be interesting to know the performance measurements if implemented in a test bed. Then the practical feasibility of LBIARM would be known.

\section{ACKNOWLEDGEMENT}

The researchers wish to thank the Deanship of Scientific Research, College of Engineering, King Saud University for supporting this research.

\section{REFERENCES}

Amir, P.A., W. Ryan, P. Marius and I. Jadwiga, 2009. ALARM: An adaptive load-aware routing metric for hybrid wireless mesh networks. Proceedings of the 32nd Australasian Computer Science Conference, Jan. 19-23, Australian Computer Society, Wellington, New Zealand, pp: 25-34.

Aiache, H., V. Conan, L. Lebrun and S. Rousseau, 2008. A load dependent metric for balancing Internet traffic in wireless mesh networks. Proceedings of the 5th IEEE International Conference on Mobile Ad Hoc and Sensor Systems, Sept. 29-Oct. 2, IEEE Xplore Press, Atlanta, GA., pp: 629-634. DOI: 10.1109/MAHSS.2008.4660098

Akyildiz, I.F., X. Wang and W. Wang, 2005. Wireless mesh networks: A survey. Comput. Netw., J., 47: 445-487. 
Alotaibi, E. and S. Roy, 2008. A location-aware routing metric (ALARM) for multi-hop, multi-channel wireless mesh networks. Proceedings of the IEEE Wireless Communications and Networking Conference, Mar. 31-Apr. 3, IEEE Xplore Press, Las Vegas, NV., pp: 2081-2086. DOI: 10.1109/WCNC.2008.369

Chu, Y. and A. Ganz, 2004. A mobile teletrauma system using $3 \mathrm{G}$ networks. IEEE Trans. Inform. Technol. Biomed., 8: 456-462. PMID: 15615036

Coury, W., J.L.R. Filho, L.A. Messina and N. Simoes, 2011. Implementing rede universitaria de telemedicina usability-the Brazilian telemedicine university network. J. Comput. Sci., 7: 1224-1229. DOI: $10.3844 /$ jcssp.2011.124.1229

Couto, D.S.J.D., D. Aguayo, J. Bicket and R. Morris, 2003. A high-throughput path metric for multi-hop wireless routing. Proceedings of the 9th Annual International Conference on Mobile Computing and Networking, Sept. 14-19, ACM Press, San Diego, CA, USA., pp: 134-146. DOI: 10.1145/938985.939000

Draves, R., J. Padhye and B. Zill, 2004. Routing in multi-radio, multi-hop wireless mesh networks. Proceedings of the 10th Annual International Conference on Mobile Computing and Networking, Sept. 26-Oct. 01, ACM Press, Philadelphia, PA, USA., pp: 114-128. DOI: 10.1145/1023720.1023732

Golmie, N., D. Cypher and O. Rebala, 2005. Performance analysis of low rate wireless technologies for medical applications. Comput. Commun., 28: 1255-1275.

Jiang, W., S. Liu, Y. Zhu and Z. Zhang, 2007. Optimizing routing metrics for large-scale multiradio mesh networks. Proceeding of the International Conference on Wireless Communications, Networking and Mobile Computing, Sept. 21-25, IEEE Xplore Press, Shanghai, pp: 1550-1553. DOI: 10.1109/WICOM.2007.390

Johnson, D.B. and D.A. Maltz, 1996. Dynamic source routing in ad hoc wireless networks. Carnegie Mellon University.

Khodambashi, R., S. Najarian, A.T. Golpaygani, A. Keshtgar and S. Torabi, 2008. A tactile sensor for detection of skin surface morphology and its application in telemedicine systems. Am. J. Applied Sci., 5: 633-638. DOI: 10.3844/ajassp.2008.633.638

Malan, D., T. Fulford-Jones, M. Welsh and S. Moulton, 2004. CodeBlue: An ad hoc sensor network infrastructure for emergency medical care. In Proceeding of the International Workshop on Wearable and Implantable Body Sensor Networks, (WIBSN' 04), London, UK.
Morreale, P.A., 2007. Wireless sensor network applications in urban telehealth. Proceeding of the 21st International Conference on Advanced Information Networking and Applications Workshops, May 21-23, IEEE Xplore Press, Niagara Falls, Ont., pp: 810-814. DOI: 10.1109/AINAW.2007.375

Perkins, C., E. Belding-Royer and S. Das, 2003. Ad hoc On-Demand Distance Vector (AODV) routing. The Internet Society.

Pradhon, A. and V. Mirchandani, 2009. Channel Assignment techniques for 802.11 based multiradio wireless mesh networks. University of Technology, Sydney.

Putra, E.H., E. Supriyanto, J. Din and H. Satria, 2009. Cross layer design of wireless lan for telemedicine application. Proceeding of the 3rd Asia International Conference on Modeling and Simulation, May 25-29, IEEE Xplore Press, Bali, pp: 264-269. DOI: 10.1109/AMS.2009.128

Shila, D.M. and T. Anjali, 2007. Load-aware traffic engineering for mesh networks. Proceedings of 16th International Conference on Computer Communications and Networks, Aug. 13-16, IEEE Xplore Press, Honolulu, HI, pp: 1040-1045. DOI: 10.1109/ICCCN.2007.4317955

Shnayder, V., B.R. Chen, K. Lorincz, T.R.F.F. Jones and M. Welsh, 2005. Sensor networks for medical care. Proceedings of the 3rd International Conference on Embedded Networked Sensor Systems, Nov. 2-4, ACM Press, San Diego, CA, USA., pp: 314-314. DOI: $10.1145 / 1098918.1098979$

Siraj, M. and K.A. Bakar, 2011a. A Load Balancing Interference Aware Routing Metric (LBIARM) for multi hop wireless mesh network. Int. J. Phys. Sci., 7: 456-461. DOI: 10.5897/IJPS11.1522

Siraj, M. and K.A. Bakar, 2011b. Link establishment and performance evaluation in IEEE 802.16 wireless mesh networks. Int. J. Phys. Sci., 6: 3189-3197.

Subramanian, A.P., M.M. Buddhikot and S. Miller, 2006. Interference aware routing in multi-radio wireless mesh networks. Proceedings of the 2nd IEEE Workshop on Wireless Mesh Networks, Sept. 25-28, IEEE Xplore Press, Reston, VA., pp: 55-63. DOI: 10.1109/WIMESH.2006.288620

Yaling, Y., J. Wang and R. Kravets, 2005. Interferenceaware load balancing for multihop wireless networks. Technical Report, University of Illinois at Urbana-Champaign.

Zhou, H., H. Ali, J.J. Youn and Z. Zhang, 2010. A hybrid wired and wireless network infrastructure to improve the productivity and quality care of critical medical applications. Proceeding of the IEEE/ICME International Conference on Complex Medical Engineering (CME), Jul. 13-15, IEEE Xplore Press, Gold Coast, QLD, pp: 215-218. DOI: 10.1109/ICCME.2010.5558842 